\title{
Research Paper: The Effectiveness of Psychoneurotherapy on Reducing Symptoms Severity in Treatment Naïve crossuark Patients With Obsessive-Compulsive Washing
}

\author{
Ali Akbar Saremi ${ }^{1}$, Mohammad Ali Nazari² ${ }^{*}$, Seyed Vahid Shariat ${ }^{3,1}$, Behrooz Dolatshahi ${ }^{4}$ \\ 1. Department of Cognitive Neuroscience, Institute for Cognitive Science Studies, Tehran, Iran \\ 2. Department of Psychology, Faculty of Education and Psychology, University of Tabriz, Tabriz, Iran. \\ 3. Department of Psychiatry, School of Behavioral Sciences \& Mental Health, Iran University of Medical Sciences, Tehran, Iran. \\ 4. Department of Clinical Psychology, University of Social Welfare and Rehabilitation Sciences, Tehran, Iran.
}

Citation: Saremi, A. A., Nazari, M. A.,Shariat, S. V., Dolatshahi, B. (2016). The Effectiveness of Psychoneurotherapy on Reducing Symptoms Severity in Treatment Naïve Patients With Obsessive-Compulsive Washing. Journal of Practice in Clinical Psychology, 4(4), 263-274. https://doi. org/10.18869/acadpub.jpcp.4.4.263

: https://doi.org/10.18869/acadpub.jpcp.4.4.263

Article info:

Received: 07 May 2016

Accepted: 01 Sep. 2016

\section{Keywords:}

Psychoneurotherapy, Obsessive-Compulsive Disorder Washing, Quantitative EEG

\begin{abstract}
Objective: The present study was carried out to examine the effectiveness of psychoneurotherapy (PNT) on reducing symptoms severity in treatment-naïve patients with obsessive-compulsive washing. This study evaluated a new form of psychotherapy based on neurobiological model of Obsessive-Compulsive Disorder Washing (OCD-W).

Methods: The study was conducted as a quasi-experimental research. The statistical population of this study included 130 obsessive-compulsive washers. A total of 40 patients were selected using convenience sampling method and then were randomly divided into two groups: PNT group $(n=20)$ and sham feedback control group $(n=20)$. Both groups received drug treatment, in addition, the experimental group received 20 sessions (twice per week) of PNT. The Yale-Brown obsessive compulsive scale (Y-BOCS), Maudsley obsessional-compulsive inventory (MOCI), depression, anxiety, and stress scale-21 (DASS-21), subjective united distress scale (SUDS) were administered before and after treatment. Eelectroencephography (EEG) was recorded in eyes open provocation stimuli condition. All EEGs were recorded drug free. Participants were prescribed PNT protocol based on the results of their quantitative EEG (QEEG) and low-resolution electromagnetic tomography analyses. The effects of PNT were analyzed using 1-way analysis of covariance (ANCOVA) after controlling pretest scores. The effect size (Cohen's d), paired t test, and clinically significant change were calculated and assessed for all the clinical instruments.
\end{abstract}

Results: Thirty patients completed this study. Despite some methodological limitations, our results indicated that psychoneurotherapy is significantly efficient on treatment's target. ANCOVA results supported that PNT could significantly improve the severity of OCD symptoms, depression, stress, anxiety symptoms, and subjective united distress scale compared to what was seen sham feedback control group (All $\mathrm{P}<0.001)$.

Conclusion: Psychoneurotherapy showed preliminarily evidence for the effectiveness in the treatment of patients with obsessive-compulsive washing. The study is an attempt to provide a new non-invasive treatment of OCD-washing. Further studies are necessary for confirming our findings.

\footnotetext{
* Corresponding Author:

Mohammad Ali Nazari, PhD

Address: Department of Psychology, Faculty of Education and Psychology, University of Tabriz, Tabriz, Iran.

Tel: +98 (51) 38833730-1

E-mail:nazaripsycho@yahoo.com
} 


\section{Introduction}

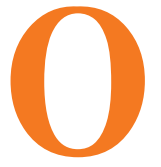

$\mathrm{CD}$ is a complex neuropsychiatric illness that is characterized by obsessions and compulsions, that the patient in order to prevent anxiety and distress or to neutralize the obsessions (American Psychiatric Association, 2013). OCD is a chronic and debilitating illness and usually causes social, family, and occupational dysfunctions (Ruscio, Stein, Chiu, \& Kessler, 2010; Taylor, 2011). OCD-washing is one of the most common ritual behaviors in Iran's clinical samples (Ghassemzadeh, Khamseh, \& Ebrahimkhani, 2005). According to Rachman (2004), there are two kinds of contamination: contact and mental. Contact contamination refers to the situation in which the people come into contact with an item that they believe may threaten their physical or mental health.

On the other hand, mental contamination happens in the absence of physical contact and refers to feelings of internal dirt and therefore urge of washing; for example, a blasphemous thought may lead to ritualistic hand washing. OCD contamination/washing subtype is distinguishable from other OCD subtypes with regard to its neuropsychological, neurobiological, behavioral, and cognitive aspects (Broderick, Grisham, \& Weidemann, 2013). Data collected from clinical fields, neuroelectrophysiology, psychosurgery, neuropharmacology, and neuroimaging research have indicated that the dysfunction of Cortico-Striato-ThalamoCortical (CSTC) circuits, play a key role in the etiology and maintenance of OCD symptoms (Menzies et al., 2008; Milad \& Rauch, 2012; Nakao, Okada, \& Kanba, 2014). OCD is associated with hypermetabolism in orbitofrontal cortex, anterior cingulate gyrus, and caudate nucleus (Del Casale et al., 2011; Harrison et al., 2009; Mataix-Cols, Lawrence, Wooderson, Speckens, \& Phillips, 2009).

Over the past two decades, the rapid development of neuroimaging techniques has provided researchers with extraordinary opportunity to study the structure and function of the brain. Among these neuroimaging techniques, we can refer to Positron Emission Tomography (PET), Magnetic Resonance Imaging (MRI), Functional magnetic Resonance Imaging (fMRI), Electroencephography (EEG), and Single-Photon Emission Computed Tomography (SPECT). Among functional neuroimaging techniques, EEG has the best temporal resolution (Coburn et al., 2006) which qualifies and analyzes the waveforms that occur on the surface of the brain and subsequently makes the analysis of human brain functions (i.e. cognition, emotion, and behavior) possible. Moreover, hospitals and private clinics can easily use it because of its conciseness and simplicity. Low-Resolution Electromagnetic Tomography (LORETA) is a modern technique to localize electrical neuronal activity of the brain detected on the scalp from EEG recordings and computes them to 3D distribution. LORETA inverse solutions provide clinical researchers with a useful and cost-effective measure (Pascual-Marqui, 2002).

Quantitative Electroencephalography (QEEG) provides some clear evidence about brain waves abnormalities in OCD. In the same vein, abnormal EEGs have been observed in patients with OCD (Kuskowski et al., 1993; Locatelli, Bellodi, Grassi, \& Scarone, 1996; Bucci et al., 2004; Pogarell et al., 2006; Velikova et al., 2010; Koprivova et al., 2011; Koprivova, Horacek, Raszka, Brunovsky, \& Prasko, 2013; Olbrich et al., 2013). Resting state EEG source localization studies reported that cingulate gyrus plays an important role in OCD (Koprivova et al., 2011; Sherlin \& Congedo, 2005; Velikova et al., 2010). LORETA technique showed an increase in low-frequency and beta EEG activity in OCD patients at rest in cingulate gyrus (Pascual-Marqui, 2002; Pascual-Marqui, Michel, \& Lehmann, 1994).

Increased resting-state beta activity in the cingulate gyrus in OCD individuals was reported (Velikova et al., 2010). Koprivova et al. (2011) found elevated lowfrequency activity $(2-6 \mathrm{~Hz})$ in the medial frontal cortex, especially anterior cingulate gyrus. Higher pretreatment delta in anterior cingulate was associated with a worse response to SSIRs (Fontenelle, Mendlowicz, Ribeiro, Piedade, \& Versiani, 2006) and neurofeedback (Koprivova et al., 2013), also higher pretreatment beta in middle of posterior cingulate gyrus was associated with a better response to neurofeedback (Koprivova et al., 2013).

Over many decades, OCD was considered one of the most resistant mental disorders to treatment. OCD treatment is unpredictable; $40 \%$ to $60 \%$ of patients have inadequate response to treatment (Pallanti \& Quercioli, 2006; Bjorgvinsoon, Hart, \& Heffelfinger, 2007). Currently, pharmacological treatment like Serotonin Reuptake Inhibitors (SRIs) and Cognitive Behavior Therapy (CBT) are the selected treatments for OCD. Potential side effects of the drug and relapse rates (24\% to $89 \%)$ are the main problems of pharmacological treatment in patients with OCD (Hollander et al., 2003; Koran, Hackett, Rubin, Wolkow, \& Robinson, 2002). Relapse rate of the disease within three months after the CBT termination was higher than 50\% (Simpson, Franklin, Cheng, Foa, \& Liebowitz, 2005; Anand, Sudhir, Math, Thennarasu, \& Reddy, 2011). Besides, psychological and pharmacological methods usually use the same treatments, regardless of the content of the obsessions (e.g. contamination, religious) and whether 
compulsions and the related rituals are overt (e.g. washing, checking) or covert (e.g. praying, counting). Therefore, more studies should be conducted on safer and more effective treatments for obsessive-compulsive washers.

Psychoneurotherapy (PNT) is based on Brain-Computer Interface (BCI) technology. It is a simple and noninvasive therapy based on clinical cognitive neuroscience to treat cognitive and emotional impairments. PNT offers the possibility to study the relation between clinical symptoms and localized brain activation. It enables us to study the influence of brain activity manipulation as an independent variable on clinical symptoms as the dependent variables (Paquettea, Beauregard, \& Prévoste, 2009). Besides, the behavioral modification caused by the operant training of localized brain areas could suggest a novel noninvasive therapeutic approach for neuropsychiatric disorders. The only study examined PNT effectiveness was Paquettea et al. (2009), which showed that PNT had been effective on the normalization of high beta brain waves $(18-30 \mathrm{~Hz})$ and on reducing the rumination, negative thoughts, anxiety, and behavioral inhibition of major depression disorder. To the best of the authors' knowledge, no study has been published on the application of PNT in OCD so far. The objective of the present study was to conduct a preliminary investigation of the effectiveness of PNT for OCD-washing, by quasi-experimental method. We hypothesized that patients would show significant reductions in OCD symptoms by receiving Psychoneurotherapy.

\section{Methods}

This research was a quasi-experimental study. Patients were recruited from the Pardis Center Clinical Psychology Service in Mashhad, Iran (from 2013 to 2015). A total of 40 patients were selected using inconvenience sampling method and then were randomly divided into two groups: PNT group $(n=20)$ and sham feedback control group $(n=20)$. PNT was applied for 20 sessions (twice per week). Both groups received drug treatment. The sham feedback group had the usual psychiatry treatments; besides they were exposed to record EEG signals of other patients 10 times during the process. All patients were treatment-naïve at the time of assessment.

The inclusion criteria adopted for this study included having OCD criteria as identified by DSM IV-IR in axis I and Y-BOCS-SR $\geq 16$ [scores are classified as mild subclinical (0-7), mild (8-15), moderate (16-23), severe (24-33), and extreme (34-40). Age between 20-45 years, holding a degree of junior high school or higher and being medication free. All subjects were right handed and with normal vision.
The patients with the history of psychotic disorders, substance or alcohol abuse, other comorbid axis I disorders, as well as medical or neurological diseases, low IQ (lower than 90), and previous treatment with psychotropic medication least 10 sessions of psychotherapy were excluded or the study. After presenting the logic of the treatment, the written consent forms were given to the patients. The study was approved by the institutional review board. To determine the sample size, a significance level of 0.05 , power of 0.08 , and an error of 0.02 were set (Cohen \& Manion, 1992). Ten $(24 \%)$ patients dropped out in process of the clinical trial ( 5 participants from the PNT and 5 from the sham feedback group), for various reasons, such as failure to comply with sessions, pregnancy, or dissatisfaction.

Participants' demographics (sex, age, education, marital status, duration of illness, previous treatment) and intelligence were recorded. For the assessment of intelligence, the Standard Progressive Matrices (SPM) (Raven, 1991) was used. The Edinburgh handedness inventory (Oldfield, 1971) was used to determine handedness.

Structured Clinical Interview for Axis I was used in this study for the diagnosis of OCD-washing (First, Spitzer, Gibbon, and Williams 2002). This interview has a good reliability for the diagnosis of mental disorders. SCID-I deals with evaluation of major mental disorders. SCID-I was used in this study for the diagnosis of OCD-washing. This study used the translated and adapted version by Sharifi et al. (2004). Diagnostic agreements of Persian version of SCID-I is above 0.60 , overall weighted $\kappa$ is 0.52 for current diagnosis, and 0.55 for all diagnosis of life-time (Sharifi et al. 2004). The other version used by this study was SCID-II, which was translated and adapted by Bakhtiari (2000). SCID-II deals with the evaluation of personality disorders (i.e. axis II in DSM IV). Bakhtiari (2000) obtained 0.95 for consistence coefficient of its testretest with one week interval.

The Yale Brown Obsessive Compulsive Scale SelfReport (Y-BOCS-SR) (Goodman et al., 1989) is usually known as the gold measure of symptom improvement in OCD treatment studies (Steketee, Frost, \& Bogart, 1996). Y-BOCS shows good reliability ( $\mathrm{a}=0.88$ to 0.91$)$ and excellent internal consistency (Goodman et al., 1989). The Iranian version (Rajezi-Esfahani, Motaghipour, AmbizKamkari, Zahiredin, \& Janbozorgi, 2012) has excellent internal consistency (Cronbach $\alpha=0.97$ ). The Y-BOCS is a 10-item self-report measure of obsessions (items 1-5) and compulsions (items 6-10). Each item is rated on a five-point Likert Scale ranging from $0(0=$ none $)$ to 4 (4=extreme symptoms). Its score ranges between 0 and 40 with a cut point score $>16$ revealing clinically important 
symptoms (storch et al., 2010). Classification of scores is as follows: subclinical (0-7), mild (8-15), moderate (16$23)$, severe (24-33), and extreme (34-40).

Depression, Anxiety and Stress Scale-21 (DASS-21)Lovibond \& Lovibond, 1995) is a self-report questionnaire with 21 items which measures symptoms of anxiety, depression, and stress. For a nonclinical sample, it has demonstrated excellent internal consistencies among its three subscales (Cronbach $\alpha=0.82-0.90$ ), good convergent and discriminating validity $(\mathrm{r}=0.70-0.72)$, and adequate reliability (Cronbach $\alpha=0.90-0.95$ ) (Henry \& Crawford, 2005). This instrument has been translated into Persian and its reliability and validity have been proved (Sahebi, Asghari, and Salari 2004).

Maudsley Obsessional-Compulsive Inventory (MOCI) is a true-false questionnaire with 30 items for the assessment of obsessive and compulsive symptoms (Hodgson \& Rachman, 1977). The MOCI has introduced as a golden measure in evaluation of treatment findings. The MOCI assesses OC symptoms across four factors: 1) washing; 2) Checking; 3) Slowness-repetition; and 4) Doubtingconscientious. Studies have demonstrated good internal consistency for the subscales, ranging from 0.60 to 0.87 (Clare, 2003). The validity and reliability have been approved by Rachman \& Hodgson (1980), and Ghassemzadeh et al. (2005). In Iran, Ghassemzadeh et al. (2005) have applied MOCI-30 in their investigations

Subjective Units of Distress Scale (SUDS) is a self-monitoring scale applied to rank distress units degree from 0 to 10. 0 represents no distress, while 10 indicates the highest level of distress (Wolpe \& Lazarus, 1966). This scale is employed in this work to determine the level of distress in participants. The presented study used this scale to determine the degree of distress among the participants.

Quantitative Electroencephalography (QEEG) was recorded in an eyes-open provocation stimuli condition, using 19 surface electrodes (Electro-Cap, Eaton, Ohio, USA) based on the international 10-20 system. The EEG was amplified by Mitsar EEG 201 System (Mitsar Medical Diagnostic Equipment, Russia). The data were recorded using a sampling rate of $250 \mathrm{~Hz}$ with a frequency band of 1 to $30 \mathrm{~Hz}$. In the present study, 19 EEG channels (Fp1, Fp2, F7, F8, F3, F4, Fz, T3, T4, C3, C4, Cz, T5, T6,Pz, $\mathrm{P} 3, \mathrm{P} 4, \mathrm{O} 1, \mathrm{O} 2)$ were used for the analysis. All electrodes were referred to linked ears. The resistance of electrodes was maintained below $5 \mathrm{k} \Omega$.

Processing of EEG signals were performed utilizing NeuroGuide Delux (version 2.3.8). In order to obtain Artifact-free epochs, they were visually analyzed by an expert. In total, 30 to 40 artifact-free EEG epochs (60 seconds) were selected for analysis. Using a Fast Fourier Transformation (FFT) algorithm, the EEG epoch time domain was converted into frequency domain. The definition of frequency bands are as follows: delta (1-4 Hz), theta $(4-8 \mathrm{~Hz})$, alpha $(8-12 \mathrm{~Hz})$ and beta $(12-30 \mathrm{~Hz})$ for which absolute and relative powers were calculated in each frequency band. By application of NeuroGuide normative database, all power values were then transformed into Z scores. Data analysis was performed by the Low Resolution Electromagnetic Tomography (LORETA) and a normative EEG database. In this study, we used the LORETA-Key software package 2003 (Key Institute for Brain-Mind Research, Zurich, Switzerland).

LORETA is a modern technique to localize electrical neuronal activity of the brain detected on the scalp from EEG recordings and computes them to $3 \mathrm{D}$ distribution. LORETA inverse solutions provide clinical studies with a useful and cost-effective measure (Pascual-Marqui, 2007). Based on LORETA, the $Z$ score of $\leq-1.5$ or $\geq 1.5$ was considered as an indicator for abnormality in score localization. Provoking EEG was recorded between 3 PM to $7 \mathrm{PM}$ for all participants in a dimly lit (approximately 40 Lux), sound attenuated room with the average temperature of $20^{\circ} \mathrm{C}$ to $23^{\circ} \mathrm{C}$. The participants were comfortably seated in a reclining chair in half lying position (approximately $45^{\circ}$ inclination). No smoking and no caffeine consumption were allowed up to 3 hours before EEG recording. According to Salinsky, Oken, and Morehead (1991) repeated samples of EEG had high reliability; at 20-seconds, 40 seconds, and 60 seconds, they were approximately $82 \%, 90 \%$, and $92 \%$ reliable respectively.

Pictures depicting contamination/washing (washing subset) were chosen from the Berlin COD-picture set (BOCD-PS) (Simon, Kischkel, Spielberg, \& Kathmann, 2012). The stimulus set has demonstrated good validity and has shown to reliably provoke symptoms in patients with OCD (Saremi et al. In Press). Twenty pictures were chosen for this dimension in the current study. The neutral pictures (NE; e.g. nature scenes) have been selected from the International Affective Picture System (IAPS) (Lang, Bradley, \& Cuthbert, 2005). The pictures were shown to 5 clinicians with experience in OCD to ensure cross cultural validation of images. In case an image was perceived as culturally inappropriate, it was replaced with a similar picture from Iranian background to increase cultural validity. Pictures that were too simple or too complex were also excluded. Stimuli were presented on a Samsung 19-inch monitor with a graphic resolution of $1024 \times 768$ pixels at a distance of approximately $70 \mathrm{~cm}$ from the participants. The trial started with a gray fixation cross $(3000 \mathrm{~ms})$ fol- 
lowed by the target pictures which lasted on the screen for $4000 \mathrm{~ms}$ and intervals of either 11000,12000 , or 13000 $\mathrm{ms}$ and ended with a white fixation cross. The pictures order was pseudo-randomized with no more than 3 pictures of each stimulus set in a row. The trial took about 9 minutes and the EEG was recorded for each patient.

Psychoneurotherapy (PNT) is based on Brain-Computer Interface $(\mathrm{BCI})$ technology. It is a clinical and noninvasive therapy based on clinical cognitive neuroscience to treat cognitive and emotional impairments. In this study, PNT was developed based on the relationship between the localization of abnormal EEG activity and OCD symptomatology. Our PNT plan for OCD comprised three stages; assessment, socialization, and intervention. The first stage included OCD-washing specific diagnostic and questionnaire, which was an OCD hierarchy creation on a scale ranging from 0 (no urge) to 9 (very strong urge), instructions to photograph personal OCD triggers and EEG recording in eyes-open condition while exposing to contamination-related stimuli from Berlin OCD (Simon et al., 2012).

The socialization stage included the treatment, informing the patient of OCD diagnosis (providing OCD booklets), presenting the logic of PNT with emphasis on EEG and cognitive emotional information processing theory (providing PNT booklets). Finally, the intervention included preparing a list of signs and symptoms which trigger obsessive thoughts (internal - external) and ritual behaviors and avoided situations, asking the subjects to take pictures of provocative stimuli of OCD personal, evaluation of subjects, case formulations, providing subjects with homework and review at the beginning of each session and allowing them to know the agenda and purpose of each session before the start of the session.

All patients received 20 sessions of PNT (60 minutes each) twice a week for 10 weeks. This study included 10 sessions of training neurofeedback based on brain waves pattern and 10 sessions of the presenting neurofeedback contamination-related animations followed by two sessions of booster. In the first ten sessions, every subject received neurofeedback training considering their QEEG pattern. Neurofeedback training which rewards or represses electrical brain activity, is a kind of operant conditioning (Demos, 2005). Each first 10 neurofeedback sessions was composed of eight to ten blocks of 3-4 minutes of active neurofeedback training separated by 1-minute breaks. During these sessions, the participants were asked to focus their visual attention on the computer screen, while the therapist motivated them to actively work on brain activity variations and associate them with their mental statues.
In the second 10 sessions of PNT, each participant was gradually exposed to individualized animations (EximiousSoft GIF Creator V7.21) based on the hierarchy of obsessions, anxiety-provoking, and avoided situations. The animations were on trigger contamination obsessions/washing compulsions in order to challenge the patients' ability to self-regulate brain activity. In intervening stage by receding brain waves from the desired target (e.g. the increase of theta waves), the animation stops moving.

To achieve the desired objectives, the patients had to change their brain waves in the determined direction. By repeating treatment sessions, the brain was gradually conditioned to make changes. Providing neurofeedback protocol was based on each individual's QEEG pattern. The PNT protocol of each participant aimed at decreasing excessive beta power at central and frontal brain areas. The most commonly trained brain areas were for monopolar: Fz, F4, F3, CZ, Fcz, Pz and for bipolar: FP1-FP2, C3-C4, F3-F4, C3-Fz.

In fact, QEEG-guided neurofeedback aimed to normalize deregulated brain regions that were related to specific clinical symptoms. Procomp Infiniti-2 software (Canadian Thought Technology Co.) was applied for neurofeedback training.

After being briefed about the research purpose, participants signed the consent forms. All participants completed a demographic questionnaire. The participants were interviewed and afterwards Y-BOCS questionnaire and MOCS, SUDs, DASS assessments and QEEG recording were undertaken before the therapy. Ten days after the last treatment session, the same assessments were conducted. It must be noted that all percipients voluntary contributed in the study and ethical considerations of the research were investigated and approved by the institutional review board (Institute for Cognitive Science Studies, Tehran, Iran). All information was kept confidential.

All statistical analyses were done using SPSS version 20 (Chicago, IL) for Windows. To determine the homogeneity of the experimental and control group, Chi-square tests and $t$ (independent) tests were used. Paired samples $t$ tests were used to compare pre-and post-treatment values for all scales on both groups. The effects of PNT were analyzed using 1-way analysis of covariance (ANCOVAs) after controlling pretest scores. The homogeneity of variance and covariance pre-assumptions were tested using Levene's test and Mauchly's test, and the assumptions were met. Effect size (Cohen's d, 1988) and mean percentage reduction $(\mathrm{MPR})(\mathrm{A} \%=\mathrm{A} 0-\mathrm{A} 1 / \mathrm{A} 0 \times 100$ : $\mathrm{A} 0=$ initial; 
A1=final) were calculated for all clinical instruments. The EEG data processed to QEEG with Neuroguide software.

\section{Results}

Demographic and clinical features of each patient are shown in Table 1. There were no significant differences between the experimental and control groups regarding age, gender, education level, IQ, marital status, age at onset of symptoms, and duration of OCD-washing. The mean age of the participants was 32.16 years $(\mathrm{SD}=6.07)$. Mean age of OCD onset was 20.67 years $(S D=3.62)$ and patients suffered from OCD for almost 11.17 years on average $(\mathrm{SD}=4.75)$. The mean Y-BOCS total score was $24.07(\mathrm{SD}=3.34)$ indicating marked psychopathology. The majority of the participants were female. The female to male ratio was 12:3 in the PNT group and 13:2 in the sham feedback group. The BDI-II scores of the OCD patients (Table 1) represent minimal severity. Scores are classified as minimal (BDI: 0-13), mild (BDI: 14-19), moderate (BDI: 20-28), or severe (BDI $>29$ ).

The mean scores and standard variations at the posttreatment stage are tabulated in Table 2. There were no significant baseline differences for mean clinical outcome measures between the PNT and sham feedback (SFB) groups (Y-BOCS total: $\mathrm{t}=-0.643, \mathrm{P}=0.526$; MOCS: $\mathrm{t}=0.174, \mathrm{P}=0.863$; DASS: $\mathrm{t}=-0.688, \mathrm{P}=0.497$; SUDS: $\mathrm{t}=0.254, \mathrm{P}=0.801)$. The results of Levene's test showed a significant $\mathrm{Y}-\mathrm{BOCS}$ total score $[\mathrm{F}(28,1)=1.13, \mathrm{P}=0.96]$.
To analyze the effect of each treatment condition on outcome measures, the paired t test was used. These results indicated a significant difference between the pretest and posttest scores of each outcome measure in the PNT and SFB conditions, meaning that PNT reduced the severity of OCD symptoms, as well as depressive, anxiety, and stress symptoms. ANCOVA was used with pretest scores as the covariate. The ANCOVA results, effect size (Cohen's d) and the mean percentage reduction of the outcome measures at the pretest and posttest points are presented in Table 3. ANCOVA results showed that PNT led to a more significant improvement in the severity of OCD symptoms, depression, stress, anxiety symptoms, and subjective united distress (All $\mathrm{P}<0.001$ ) than sham feedback control group. After treatment, symptoms were significantly reduced regarding Y-BOCS $(\mathrm{PNT}=52.97 \%, \mathrm{SFB}=29.17 \%)$, MOCS $(\mathrm{PNT}=59.88 \%$, $\mathrm{SFB}=22.37 \%)$, SUDS (PNT $=43.85 \%, \quad \mathrm{SFB}=19.25 \%)$, and DASS $(\mathrm{PNT}=47.97 \%, \mathrm{SFB}=36.43 \%)$. The effect size (Cohen's d) between initial and final Y-BOCS $(\mathrm{PNT}=2.67, \mathrm{SFB}=1.77), \mathrm{MOCS}(\mathrm{PNT}=1.94, \mathrm{SFB}=0.72)$, SUDS (PNT=1.15, SFB=0.55), and DASS (PNT=2.72, $\mathrm{SFB}=2.63$ ) with significant statistical difference between treatments. Large effect sizes were observed for differences between the PNT and SFB groups.

\section{Discussion}

Clinical, epidemiological, neuropsychological, and intervention studies have demonstrated the heterogeneous nature of OCD. This study was mainly aimed to find an

Table 1. Demographic and clinical characteristics of the participants.

\begin{tabular}{ccccccc}
\hline \multirow{2}{*}{ Variable } & \multicolumn{2}{c}{ PNT=15 } & \multicolumn{2}{c}{ SFB=15 } & T/ $\chi^{2}$ & Sig. \\
\cline { 2 - 7 } & Mean & SD & Mean & SD & & \\
\hline Age (y) & 31.53 & 5.37 & 32.80 & 6.78 & -0.567 & 0.57 \\
Gender (female/male) & & $12: 3$ & & $13: 2$ & $\chi^{2}(0.42)$ & 0.62 \\
Marital status (single/married) & & $6: 9$ & & $4: 11$ & $\chi^{2}(0.85)$ & 0.72 \\
Education (y) & 14.33 & 3.41 & 14.60 & 2.69 & -0.237 & 0.81 \\
Age at onset of symptoms & 20.07 & 3.73 & 21.27 & 3.51 & -0.907 & 0.37 \\
Duration of symptoms (y) & 11.27 & 4.68 & 11.07 & 4.83 & 0.115 & 0.90 \\
\hline IQ (general) & 111.07 & 5.02 & 112.33 & 3.18 & -0.826 & 0.416 \\
BDI-II & 13.20 & 1.70 & 12.93 & 1.71 & 0.428 & 0.672 \\
\hline
\end{tabular}

Abbreviations: PNT: Psychoneurotherapy; SFB: Sham Feedback; SD: Standard Deviation; IQ: Intelligence Quotient; BDI-II: Beck Depression Inventory- Second Edition. 
Table 2. Means, standard deviations and paired t results for the outcome measures.

\begin{tabular}{|c|c|c|c|c|c|c|c|c|c|c|c|c|}
\hline \multirow{3}{*}{$\begin{array}{c}\text { Groups } \\
\text { Stages } \\
\text { Measures }\end{array}$} & \multicolumn{6}{|c|}{ PNT=15 } & \multicolumn{6}{|c|}{$S F B=15$} \\
\hline & \multicolumn{2}{|c|}{ Pretest } & \multicolumn{2}{|c|}{ Posttest } & \multirow{2}{*}{$\begin{array}{l}\text { Paired } \\
t=(14)\end{array}$} & \multirow{2}{*}{$\mathbf{P}$} & \multicolumn{2}{|c|}{ Pretest } & \multicolumn{2}{|c|}{ Posttest } & \multirow{2}{*}{$\begin{array}{l}\text { Paired } \\
t=(14)\end{array}$} & \multirow{2}{*}{$\mathbf{P}$} \\
\hline & Mean & SD & Mean & SD & & & Mean & SD & Mean & SD & & \\
\hline Y-BOCS & 23.67 & 5.01 & 11.13 & 4.40 & 9.912 & 0.000 & 24.47 & 3.67 & 17.33 & 4.37 & 7.389 & 0.000 \\
\hline MOCS & 8.80 & 3.18 & 3.53 & 2.13 & 9.471 & 0.000 & 8.67 & 3.02 & 6.73 & 2.28 & 3.194 & 0.006 \\
\hline SUDS & 7.00 & 2.69 & 3.93 & 2.62 & 6.227 & 0.000 & 7.27 & 2.79 & 5.87 & 2.25 & 2.881 & 0.012 \\
\hline DASS & 67.27 & 7.33 & 35.00 & 12.13 & 32.663 & 0.000 & 68.07 & 7.03 & 43.27 & 11.28 & 19.892 & 0.000 \\
\hline
\end{tabular}

Abbreviations: Y-BOCS: Yale Brown Obsessive Compulsive Scale; MOCS: Maudsley Obsessional-Compulsive Inventory; SUDS: Subjective Units of Distress Scale; DASS: Depression, Anxiety, and Stress Scale.

Table 3. Mean percentage reduction, effect size (Cohen's d), and ANCOVA results for the outcome measures.

\begin{tabular}{ccccccccc}
\hline & \multicolumn{2}{c}{ MPR } & \multicolumn{2}{c}{ Effect Size (Cohen's d) } & \multicolumn{3}{c}{ ANCOVA } \\
\hline Groups & PNT & SFB & PNT & SFB & F & P & $\eta^{2}$ \\
Measures & & & & & & & & \\
\hline Y-BOCS & 52.97 & 29.17 & 2.76 & 1.77 & 23.559 & 0.000 & 0.997 \\
MOCS & 59.88 & 22.37 & 1.94 & 0.72 & 52.651 & 0.000 & 1.000 \\
SUDS & 43.85 & 19.25 & 1.15 & 0.55 & 13.165 & 0.001 & 0.938 \\
DASS & 47.97 & 36.43 & 2.72 & 2.63 & 21.453 & 0.000 & 0.994 \\
\hline
\end{tabular}

Abbreviations: MPR: Mean Percentage Reduction; ANCOVA: Analysis of Covariance.

answer to this question; "Is PNT an effective treatment for OCD-washing?". This was the first study in Iran addressing effectiveness of PNT for OCD-washing. Both treatments (PNT and SFB) were shown to be effective in reducing obsessive-compulsive symptoms. Findings have indicated a reduction of the obsessive-compulsive symptoms severity in PNT group. The improvement percentage of the obsession-compulsion scores was significantly greater in the PNT group compared to sham feedback group.

The only study examining PNT effectiveness was Paquettea et al. (2009), which indicated that PNT was effective on the normalization of high beta brain waves $(18-30 \mathrm{~Hz})$ and reducing the rumination, negative thoughts, anxiety, and behavioral inhibition of major depression disorder. The present study findings are in line with previous research in effectiveness of neurofeedback on improving the OCD symptoms (Hammond, 2003, 2004; Lazarov, Dar, \& Oded, 2010; Ye, An \& Wang, 2010; Koprivova et al., 2013; Deng et al., 2014). In this study, patients with OCD showed sig- nificantly reduced symptoms after treatment, with a large effect size. The average Y-BOCS for PNT recovered or improved at post-treatment was $12.53(\mathrm{SD}=4.90)$.

According to Fisher and Wells (2005), a cutoff score of 14 on the Y-BOCS is considered as a clinically significant change and of 10 as a reliable change. Rufer, Fricke, Moritz, Kloss, and Hand (2006) stated that regarding YBOCS score, patients with a decrease of $\geq 35 \%$ may be classified as responders while those with a decrease of $35 \%$ can be classified as non-responders. Patients with OCD demonstrate significant reduction in cerebral metabolism in the orbitofrontal cortex, anterior cingulate cortex, caudate nucleus, cerebellum, and thalamus post-treatment (Saxena et al., 1999, 2009; Schwartz et al., 1996).

Previous before and after treatment Comparative studies facilitate the estimation of correlation between changes of brain function and clinical symptoms improvement for both pharmacological and psychological treatments 
(Baxter et al., 1992; Saxena et al., 1999, 2009; Schwartz et al., 1996; Nakao et al., 2005; Yamanishi et al., 2009; Apostolova et al., 2010). Moderate to large effect sizes were reported by Lack and Storch (2008), when applying computer-administered CBT for OCD. Tot, Ozge, Comelekoglu, Yazici, and Bal (2002) reported decreased beta activity in left frontal during hyperventilation for OCD treatment responders.

QEEG research suggests that individuals with OCD show a different pattern of brain waves activity. An increase in alpha and beta waves (Velikova et al., 2010; Bolwig, Hansen, Hansen, Merkin, \& Prichep, 2007), decrease in alpha and beta waves (Bucci et al., 2004; Serra, Palma, Nolfe, \& Buscaino, 1994) and delta or theta waves increase (Koprivova et al., 2011; Velikova et al., 2010; Pogarell et al., 2006; Karadag et al., 2003; Tot, Ozge, Comelekoglu, Yazici, \& Bal, 2002; Simpson, Tenke, Towey, Liebowitz, \& Bruder, 2000) were reported in OCD patients. For instance, the results of Bucci et al., (2004) suggests a relative decrease of alpha band activity among OCD patients before treatment, which was then normalized following the treatment. Patients with OCD have shown an increase in the theta band at frontotemporal regions (Karadag et al., 2003).

The results of different EEG studies in OCD patients have so far been mostly inconsistent. The reason for these inconsistencies is varied and includes lack of a control group, difference in EEG methods, medication differences, and heterogeneity of the samples. Based on neuroimaging data, Nakao et al. (2005) reported that CBT changed brain activity in patients with OCD when responding to symptom provocation and neuropsychological tasks. Based on the QEEG, Prichep et al. (1993) diagnosed two clusters of OCD: cluster 1 included excess relative power in theta (on frontal and fronto-temporal areas) and cluster 2 included excess relative power in alpha (especially on T5, P3, O1 areas), which responded differently to SRIs. While $20 \%$ of cluster 1 showed a positive response to SRIs, $82.4 \%$ of cluster 2 showed a positive response to SRIs. A case study conducted by Hammond (2003) and (2004) has reported the effect of neurofeedback training on the improvement of OCD symptoms.

In a study conducted by Koprivova et al. (2013), 20 patients with OCD were selected and randomly divided into two groups, neurofeedback $(\mathrm{n}=10)$ and sham feedback $(n=10)$. The treatment protocol was based on the QEEG findings. The results indicated that the reduction percentage of compulsive behaviors of neurofeedback group was significant compared to feedback sham group, but there was not a significant difference in the obsessive thoughts, depression, and anxiety scales between two groups. Besides, after the treatment, a decrease of the relative alpha1 power (8-10) was reported on posterior cingulate gyrus and also a decrease of absolute beta3 power.

Baioui et al. (2013) showed that after improving clinical symptoms of obsessive-compulsive washers with CBT, the hyperactivity of frontostriatal circuit reduced. Furthermore after the treatment, the analysis of fMRI results of OCD patients showed a reduction in the nucleus accumbens and posterior supramarginal gyrus activities during symptoms provocation of individualized stimulus OCD (washing/contamination) and a reduction in the orbitofrontal cortex, nucleus caudatus, ventrolateral prefrontal cortex and anterior supramarginal gyrus activities during symptoms provocation of standardized Maudsley Obsessive Compulsive Stimuli Set (MOCSS). This confirms the validity of symptoms provocation.

A neuroscientific review study for OCD by Nakao et al. (2014) has reported that CBT reduced compulsive behaviors and improved cognition by acting on the cortical regions, (especially the dorsolateral prefrontal cortex). Also selective serotonin reuptake inhibitors and neuroleptics cause the emotions improvement and reduction of obsessions by acting on the limbic system and the basal ganglia areas. Clinical expertise often turn toward strategies that enhance the therapeutic effect thereby they can optimize the improvement rate of OCD symptoms. Deng et al. (2014) demonstrated that the neurofeedback adjunctive with CBT and pharmacological treatment not only reduced OCD symptoms, but also improved neurocognitive functions.

Review of previous researches reveals lack of the studies focused on efficacy of PNT for OCD patients. However, in accordance with the result of the present study, there are some studies which show the effectiveness of CBT on OCD treatment (Otte, 2011; Olatunji, Cisler, \& Deacon, 2010). Although neuroimaging studies of OCD psychotherapy are limited, some research on the effects of psychotherapy on brain structure and function have had promising results. Psychotherapy decreases the hyperactivity of brain regions that are associated with compulsive behaviors (Nakao et al., 2014), also decreases the activity in the amygdalohippocampal subcortical region (i.e. bottom-up processing), and increases the activity in the frontal cortical regions (i.e. top-down processing) (Clark \& Beck, 2010). Finally, in explaining the results obtained, we can argue that the changes in overt behaviors are in fact a reflection of the changes in the brain (covert behavior).

Attempts to change brain waves through methods such as PNT lead to changes in the brain functioning. Any 
changes following the treatment in the electrical activity of the brain reorganize the whole electrophysiological system. This reorganization creates a comprehensive normalization, reflective and natural reaction on the brain that leads to improvement. Therefore, EEG changes are likely the results of complex reorganization of EEG activity. As a result, PNT by modifying abnormal brain waves can be considered as one treatment option for patients with OCD. The most important point of this study is the application of psychoneurotherapy method based on the clinical cognitive neuroscience for the first time in Iran.

Patient-specific treatment protocols could enhance the effectiveness of PNT. However, one of the limitations of this paper was that such treatment protocol was designed and applied for the first time on OCD-washing in Iran, the fore restricting generalization of these results. Moreover as the sample size of this study was relatively small selected via convenient sampling, it is recommended to apply random sampling and consider larger sample sizes in future works in order to be able to generalize the results. Also, the sample included specific, highly educated people, so the findings might be limited to this group people. The clinical variables of this study were based on dimensional self-report questionnaires, which limit their validity. Also as OCD-washing patients with no other comorbid conditions were the subject of present study, results could not be generalize for the all OCD-washing patient who suffer from the comorbid conditions. Also, future studies have better to investigate the rate of recovery and improvement at short- and long-term follow-ups. The comparison between the clinical impacts of PNT and those of traditional treatments for OCD is also of crucial importance.

In conclusion, PNT is a non-invasive treatment which could improve the obsessive-compulsive, depressive, and anxiety symptoms effectively in patients with OCD. PNT could adjust the patient's abnormal brain electrical activity resulting in improving clinical OCD symptoms. This is the first study carried out in Iran, which adopted PNT in the management of patients with OCD-washing. The results of this preliminary investigation focused on more controlled assessment researches addressing effectiveness of OCD are necessary. The study has some significant implications.

\section{Acknowledgements}

The authors truly appreciate all the participants and colleagues who helped us in this study. The study was not sponsored by any grant. This article was part of the first author's Ph.D. thesis.

\section{Conflict of Interest}

The authors did not have any conflict of interests to report.

\section{References}

Apostolova, I., Block, S., Buchert, R., Osen, B., Conradi, M., Tabrizian, S., et al. (2010). Effects of behavioral therapy or pharmacotherapy on brain glucose metabolism in subjects with obsessive-compulsive disorder as assessed by brain FDG PET. Psychiatry Research: Neuroimaging, 184(2), 105-116. doi: 10.1016/j.pscychresns.2010.08.012

American Psychiatric Association. (2013). Diagnostic and statistical manual of mental disorders: DSM-5 ( $5^{\text {th }}$ ed.). Arlington, V. A. American Psychiatric Association.

Anand, N., Sudhir, P. M., Math, S. B., Thennarasu, K., \& Reddy, Y. C. (2011). Cognitive behavior therapy in medication nonresponders with obsessive-compulsive disorder: a prospective 1-year follow-up study. Journal of Anxiety Disorders, 25(7), 939-45. doi: 10.1016/j.janxdis.2011.05.007

Baioui, A., Pilgramm, J., Kagerer, S., Walter, B., Vaitl, D., \& Stark, R. (2013). Neural correlates of symptom reduction after CBT in obsessive-compulsive washers: An fMRI symptom provocation study. Journal of Obsessive-Compulsive \& Related Disorders, 2(3), 322-30. doi: 10.1016/j.jocrd.2013.04.006

Baioui, A., Pilgramm, J., Merz, C. J., Walter, B., Vaitl, D., \& Stark, R. (2013). Neural response in obsessive-compulsive washers depends on individual fit of triggers. Frontiers in Human Neuroscience, 7, 143. doi: 10.3389/fnhum.2013.00143

Baxter Jr., L. R., Schwartz, J. M., Bergman, K. S., Szuba, M. P., Guze, B. H., Mazziotta, J. C., et al. (1992). Caudate glucose metabolic rate changes with both drug and behavior therapy for obsessive-compulsive disorder. Archives of General Psychiatry, 49(9), 681-89. doi: 10.1001/archpsyc.1992.01820090009002

Bakhtiari, M. (2000). [The study of mental disorders in persons with body dysmorphic disorders (Persian)] (MA thesis). Tehran: Iran University of Medical Sciences.

Berenbaum, H. \& Prince, J. D. (1994). Alexithymia and the interpretation of emotion-relevant information. Cognitive Emotion, 8, 231-244.

Bjorgvinsson, T., Hart, J., \& Heffelfinger, S. (2007). Obsessivecompulsive disorder: Update on assessment and treatment. Journal of Psychiatric Practice, 13(6), 362-72. doi: 10.1097/01. pra.0000300122.76322.ad

Bolwig, T. G., Hansen, E. S., Hansen, A., Merkin, H., \& Prichep, L. S. (2007). Toward a better understanding of the pathophysiology of OCD SSRI responders: QEEG source localization. Acta Psychiatrica Scandinavica, 115(3), 237-42. doi: 10.1111/j.16000447.2006.00889.x

Broderick, J., Grisham, J., \& Weidemann, G. (2013). Disgust and fear responding in contamination-based obsessive-compulsive disorder during pictorial exposure. Behavior Therapy, 44(1), 27-38. doi: 10.1016/j.beth.2012.05.005 
Bucci, P., Mucci, A., Volpe, U., Merlotti, E., Galderisi, S., \& Maj, M. (2004). Executive hyper control in obsessive compulsive disorder: Electrophysiological and neuropsychological indices. Clinical Neurophysiology, 115(6), 1340-348. doi: 10.1016/j. clinph.2003.12.031

Clare, T. S. (2003). Assessment Procedures, In R. G. Menzies \& P. De Silva (Eds.), Obsessive-Compulsive Disorder: Theory Research and Treatment (pp. 239-58). Chichester: John Wiley.

Clark, D. A., \& Beck, A. T. (2010). Cognitive theory and therapy of anxiety and depression: Convergence with neurobiological findings. Trends in Cognitive Sciences, 14(9), 418-24. doi: 10.1016/j.tics.2010.06.007

Coburn, K. L., Lauterbach, E. C., Boutros, N. N., Black, K. J., Arciniegas, D. B., \& Coffey, C. E. (2006). The value of quantitative electroencephalography in clinical psychiatry: A report by the Committee on Research of the American Neuropsychiatric Association. Journal of Neuropsychiatry and Clinical Neuroscience, 18(4), 460-500. doi: 10.1176/jnp.2006.18.4.460

Cohen, J. (1988). Statistical power analysis for the behavioral sciences $\left(2^{\text {nd }}\right.$ ed.). Hillsdale, N. J.: Erlbaum.

Cohen, L., \& Manion, L. (1992). Research methods in education. London: Routledge.

Del Casale, A., Kotzalidis, G. D., Rapinesi, C., Serata, D., Ambrosi, E., Simonetti, A., et al. (2011). Functional neuroimaging in obsessive-compulsive disorder. Neuropsychobiology, 64(2), 61-85. doi: $10.1159 / 000325223$

Demos, J. N. (2005). Getting started with neurofeedback. New York, N. Y.: W.W. Norton \& Company Inc.

Deng, X., Wang, G., Zhou, X., Yang, M., Han, G., TU, Z., et al. (2014). Randomized controlled trial adjunctive EEG-biofeedback treatment of obsessive-compulsive disorder. Shanghai Archive Psychiatry, 26(5), 272-79. doi:10.11919/j.issn.10020829.214067

First, R., Spitzer, R., Gibbon, F., \& Williams, H. (1989). Structured Clinical Interview for DSM-IVTR [P. Mohamadkhani, O. Jahantabesh \& Sh. Tamanaeifar (Persian trans.)]. Tehran: Danjhe Publishing.

First, M., Gibbon, M., \& Spitzer, R. (2002). User's guide for SCID-I Structured Clinical Interview for DSM-IV-TR Axis I Disorders, research version. New York: New York State Psychiatric Institute.

First, M. B., Spitzer, R. L., Gibbon, M., \& Williams, J. B. (2002). Structured clinical interview for DSM-IV-TR Axis I Disorders, Research Version, Patient Edition (SCID-I/P). New York: New York State Psychiatric Institute.

Fisher, P. L., \& Wells, A. (2005). How effective are cognitive and behavioral treatments for obsessive-compulsive disorder? A clinical significance analysis. Behaviour Research and Therapy, 43(12), 1543-558. doi: 10.1016/j.brat.2004.11.007

Fontenelle, L. F., Mendlowicz, M. V., Ribeiro, P., Piedade, R. A., \& Versiani, M. (2006). Low-resolution electromagnetic tomography and treatment response in obsessive-compulsive disorder. International Journal of Neuropsychopharmacology, 9(1), 89-94. doi: 10.1017/S1461145705005584

Ghassemzadeh, H., Khamseh, A., \& Ebrahimkhani, N. (2005). Demographic Variables and Clinical Features of Obsessive-Compulsive Disorder in Iranian Patients (Second Report). In B. E.
Ling (ed.), Obsessive-Compulsive Disorder Research (pp. 241-71) New York: Nova Science Publishers, Inc.

Goodman, W. K., Price, L. H., Rasmussen, S. A., Mazure, C., Fleischmann, R. L., \& Hill, C. L. (1989). The Yale-Brown Obsessive Compulsive Scale (Y-BOCS) I. Development, use and reliability. Archives of General Psychiatry, 46(11), 1006-011. PMID: 2684084

Hammond, D. C. (2003). QEEG-guided neurofeedback in the treatment of obsessive-compulsive disorder. Journal of Neurotherapy, 7(2), 25-52. doi: 10.1300/j184v07n02_03

Hammond, D. C. (2004). Treatment of obsessional subtype of OCD with neurofeedback. Biofeedback, 32, 9-12.

Hammond, D. C. (2005). Neurofeedback treatment of depression and anxiety. Journal of Adult Development, 12(2, 3), 131-38. doi: 10.1007/s10804-005-7029-5

Harrison, B. J., Soriano-Mas, C., Pujol, J., Ortiz, H., Lopez-Sola, M., \& Hernandez-Ribas, R. (2009). Altered corticostriatal functional connectivity in obsessive-compulsive disorder. Archives of General Psychiatry, 66(11), 1189-200. doi: 10.1001/archgenpsychiatry.2009.152

Henry, J. D., \& Crawford, J. R. (2005). The short-form version of the Depression Anxiety Stress Scales (DASS-21): Construct validity and normative data in a large non-clinical sample. British Journal of Clinical Psychology, 44(2), 227-39. doi: 10.1348/014466505X29657

Hodgson, R. J., \& Rachman, S. (1977). Obsessional-compulsive complaints. Behaviour Research and Therapy, 15(5), 389-95. doi: 10.1016/0005-7967(77)90042-0

Hollander, E., Allen, A., Steiner, M., Wheadon, D. E., Oakes, R., \& Burnham, D. B. (2003). Acute and long-term treatment and prevention of relapse of obsessive-compulsive disorder with paroxetine. Journal of Clinical Psychiatry, 64(9), 1113-121. doi: 10.4088/jcp.v64n0919

Karadag, F., Oguzhanoglu, N. K., Kurt, T., Oguzhanoglu, A., Atesci, F., \& Ozdel, O. (2003). Quantitative EEG analysis in obsessive compulsive disorder. International Journal of Neuroscience, 113(6), 833-47. doi: 10.1080/00207450390200963

Koprivova, J., Congedo, M., Horacek, J., Prasko, J., Raszka, M., Brunovsky, M., et al. (2011). EEG source analysis in obsessivecompulsive disorder. Clinical Neurophysiology, 122(9), 1735-743. doi: 10.1016/j.clinph.2011.01.051

Koprivova, J., Horacek, J., Raszka, M., Brunovsky, M., \& Prasko, J. (2013). Standardized low-resolution electromagnetic tomography in obsessive-compulsive disorder a replication study. Neuroscience Letters, 548, 185-89. doi: 10.1016/j.neulet.2013.05.015

Koprivova, J., Congedo, M., Razska, M., Prasko, J., Brunovsky, M., \& Horacek, J. (2013). Prediction of treatment response and the effect of independent component neurofeedback in obsessive compulsive disorder: A randomized, sham-controlled, and double-blind study. Neuropsychobiology, 67(4), 210-23. doi: $10.1159 / 000347087$

Koran, L. M., Hackett, E., Rubin, A., Wolkow, R., \& Robinson, D. (2002). Efficacy of sertraline in the long-term treatment of obsessive-compulsive disorder. American Journal of Psychiatry, 159(1), 88-95. doi: 10.1176/appi.ajp.159.1.88

Kuskowski, M. A., Malone, S. M., Kim, S. W., Dysken, M. W., Okaya, A. J., \& Christensen, K. J. (1993). Quantitative EEG in 
obsessive-compulsive disorder. Biological Psychiatry, 33(6), 42330. PMID: 8490069

Lack, C. W., \& Storch, E. A. (2008). The use of computers in the assessment and treatment of obsessive-compulsive disorder. Computers in Human Behavior, 24(3), 917-29. doi: 10.1016/j. chb.2007.02.010

Lang, P. J., Bradley, M. M., \& Cuthbert, B. N. (2005). International affective picture system (IAPS): Affective ratings of pictures and instruction manual. Technical report A-6. Gainesville, F. L.: University of Florida.

Lazarov, A., Dar, R., \& Oded, Y. (2010). Are obsessive compulsive tendencies related to reliance on external proxies for internal states evidence from biofeedback-aided relaxation studies? Behaviour Research \& Therapy, 48(6), 516-23. doi: 10.1016/j. brat.2010.02.007

Locatelli, M., Bellodi, L., Grassi, B., \& Scarone, S. (1996). EEG power modifications in obsessive-compulsive disorder during olfactory stimulation. Biological Psychiatry, 39(5), 326-31. doi: 10.1016/0006-3223(95)00172-7

Lovibond, S. M., \& Lovibond, P. F. M. (1995). Manual of depressionanxiety-stress scale. Australia: Psychology Foundation of Australia Inc.

Mataix-Cols, D., Lawrence, N. S., Wooderson, S., Speckens, A., \& Phillips, M. L. (2009). The Maudsley obsessive-compulsive stimuli set: Validation of a standardized paradigm for symptom-specific provocation in obsessive-compulsive disorder. Psychiatry Research: Neuroimaging, 168(3), 238-41. doi:10.1016/j. psychres.2008.05.007

Matsunaga, H., Hayashida, K., Kiriike, N., Maebayashi, K., \& Stein, D. (2010). The clinical utility of symptom dimensions in obsessive-compulsive disorder. Psychiatry Research: Neuroimaging, 180(1), 25-29. doi: 10.1016/j.psychres.2009.09.005

Menzies, L., Chamberlain, R., Laird, A. R., Thelen, S. M., Sahakian, B. J., \& Bullmore, E. T. (2008). Integrating evidence from neuroimaging and neuropsychological studies of obsessivecompulsive disorder: The orbitofronto-striatal model revisited. Neuroscience \& Biobehavioral Reviews, 32(3), 525-49. doi: 10.1016/j.neubiorev.2007.09.005

Meyers, L. S., Gamst, G., \& Guarino, A. J. (2006). Applied multivariate research: design and interpretation. Thousand Oaks: Sage Publication India.

Milad, M. R., \& Rauch, S. L. (2012). Obsessive-compulsive disorder: beyond segregated cortico-striatal pathway. Trends in Cognitive Sciences, 16(1), 43-51. doi: 10.1016/j.tics.2011.11.003

Nakao, T., Nakagawa, A., Yoshiura, T., Nakatani, E., Nabeyama, M., Yoshizato, C., et al. (2005). A functional MRI comparison of patients with obsessive-compulsive disorder and normal controls during a Chinese character Stroop task. Psychiatry Research: Neuroimaging, 139(2), 101-14. doi: 10.1016/j.pscychresns.2004.12.004

Nakao, T., Okada, K., \& Kanba, S. H. (2014). Neurobiological model of obsessive-compulsive disorder: Evidence from recent neuropsychological and neuroimaging findings. Psychiatry and Clinical Neurosciences, 68(8), 587-605. doi: 10.1111/pcn.12195

Olatunji, B. O., Cisler, J. M., \& Deacon, B. J. (2010). Efficacy of cognitive behavioral therapy for anxiety disorders: A review of meta-analytic findings. Psychiatric Clinics of North America, 33(3), 557-77. doi: 10.1016/j.psc.2010.04.002
Olbrich, S., Olbrich, H., Adamaszek, M., Jahn, I., Hegerl, U., \& Stengler, K. (2013). Altered EEG lagged coherence during rest in obsessive-compulsive disorder. Clinical Neurophysiology. doi: 10.1016/j.clinph.2013.05.031

Oldfield, R. C. (1971). The assessment and analysis of handedness: The Edinburgh inventory. Neropsychologia, 9(1), 97-113. doi: 10.1016/0028-3932(71)90067-4

Otte, C. (2011). Cognitive behavioral therapy in anxiety disorders: current state of the evidence. Dialogues in Clinical Neuroscience, 13(4), 413-21. PMCID: PMC3263389

Pallanti, S., \& Quercioli, L. (2006). Treatment-refractory obsessive-compulsive disorder: methodological issues, operational definitions and therapeutic lines. Progress in Neuropsychopharmacology \& Biological Psychiatry, 30(3), 400-12. doi: 10.1016/j. pnpbp.2005.11.028

Paquettea, V., Beauregard, M., \& Prévoste, D. B. (2009). Effect of a psychoneurotherapy on brain electromagnetic tomography in individuals with major depressive disorder. Psychiatry Research: Neuroimaging, 174(3), 231-39. doi: 10.1016/j.pscychresns.2009.06.002

Pascual-Marqui, R. D. (2007). LORETA: Low resolution brain electromagnetic tomography. Zurich: The KEY Institute for BrainMind Research

Pascual-Marqui, R. D. (2002). Standardized low-resolution brain electromagnetic tomography (sLORETA): Technical details. Methods and Findings in Experimental and Clinical Pharmacology, 24, 5-12. PMID: 12575463

Pascual-Marqui, R. D., Michel, C. M., \& Lehmann, D. (1994). Low resolution electromagnetic tomography: a new method for localizing electrical activity in the brain. International Journal of Psychophysiology, 18(1), 49-65. doi: 10.1016/01678760(84)90014-x

Pogarell, O., Juckel, G., Mavrogiorgou, P., Mulert, C., Folkerts, M., Hauke, W., et al. (2006). Symptom-specific EEG power correlations in patients with obsessive-compulsive disorder. International Journal of Psychophysiology, 62(1), 87-92. doi: 10.1016/j. ijpsycho.2006.02.002

Prichep, L. S., Mas, F., Hollander, E., Liebowitz, M., John, E. R., Almas, M., et al. (1993). Quantitative electroencephalographic sub typing of obsessive-compulsive disorder. Psychiatry Research: Neuroimaging, 50(1), 25-32. doi: 10.1016/0925-4927(93)90021-9

Rachman, S. (2004). Fear of contamination. Behavior Research \& Therapy, 42(11), 1227-255. doi: 10.1016/j.brat.2003.10.009

Rachman, S. J., \& Hodgson, R. J. (1980). Obsessions and compulsions. Englewood Cliffs, N. J.: Prentice Hall.

Rajezi Esfahani, S., Motaghipour, Y., AmbizKamkari, K., Zahiredin, A., \& Janbozorgi, M. (2012). [Reliability and validity of the Persian version of the Yale-Brown obsessive-compulsive scale (YBOCS) (Persian)]. Iranian Journal of Psychiatry and Clinical Psychology, 17(4), 297-303.

Raven, J. (1991). Manual for Raven's progressive matrices and Mill Hill vocabulary scales. Oxford: Oxford Psychologists Press.

Rufer, M., Fricke, S., Moritz, S., Kloss, M., \& Hand, I. (2006). Symptom dimensions in obsessive-compulsive disorder: prediction of cognitive-behavior therapy outcome. Acta Psychiatrica Scandinavica, 113(5), 440-46. doi: 10.1111/j.1600-0447.2005.00682.x 
Ruscio, A. M., Stein, D. J., Chiu, W. T., \& Kessler, R. C. (2010). The epidemiology of obsessive-compulsive disorder in the National Comorbidity survey replication. Molecular Psychiatry, 15(1), 53-63. doi: $10.1038 / \mathrm{mp} .2008 .94$

Sahebi, A., Asghari, M. J., \& Salari, R. S. (2004). [Validation of depression anxiety and stress scale (DASS-21) for an Iranian population (Persian)]. Journal of Developmental Psychology, 4(1), 299-313.

Salinsky, M. C., Oken, B. S., \& Morehead, L. (1991). Test-retest reliability in EEG frequency analysis. Electroencephalography and Clinical Neurophysiology, 79(5), 383-92. doi: 10.1016/00134694(91)90203-g

Saremi, A. A. [The effectiveness of a psychoneurotherapy on neuroelectrophysiological, neuropsychological functioning's and clinical symptoms in individuals with obsessive - compulsive disorder washing (Persian)] [PhD thesis]. Tehran: Institute for Cognitive Science Studies; 2016.

Saxena, S., Gorbis, E., O'Neill, J., Baker, S. K., Mandelkern, M. A., Maidment, K. M., et al. (2009). Rapid effects of brief intensive cognitive-behavioral therapy on brain glucose metabolism in obsessive-compulsive disorder. Molecular Psychiatry, 14(2), 197-205. doi: 10.1038/sj.mp.4002134

Saxena, S., Brody, A. L., Maidment, K. M., Dunkin, J. J., Colgan, M., Alborzian, S., et al. (1999). Localized orbitofrontal and subcortical metabolic changes and predictors of response to paroxetine treatment in obsessive-compulsive disorder. Neuropsychopharmacology, 21(6), 683-93. doi: 10.1016/s0893$133 \times(99) 00082-2$

Schwartz, J. M., Stoessel, P. W., Baxter Jr., L. R., Martin, K. M., \& Phelps, M. E. (1996). Systematic changes in cerebral glucose metabolic rate after successful behavior modification treatment of obsessive-compulsive disorder. Archives of General Psychiatry, 53(2), 109-13. doi: 10.1001/archpsyc.1996.01830020023004

Serra, F. P., Palma, V., Nolfe, G., \& Buscaino, G. A. (1994). An electrophysiological study in obsessional compulsive disorders. Acta Neurologica, 16(5, 6), 240-48. PMID: 7709794

Sharifi, V., Asaadi, M., Mohammadi, M., Amini, H., Kaviani, H., Semnani, Y., et al. (2004). [Reliability and feasibility of the Persian version of the structured diagnostic interview for DSM-IV (SCID) (Persian)]. Advances in Cognitive Science, 6(1-2), 10-22.

Sherlin, L., \& Congedo, M. (2005). Obsessive-compulsive dimension localized using low resolution brain electromagnetic tomography (LORETA). Neuroscience Letters, 387(2), 72-74. doi: 10.1016/j.neulet.2005.06.069

Simon, D., Kischkel, E., Spielberg, R., \& Kathmann, N. (2012). A pilot study on the validity of using pictures and videos for individualized symptom provocation in obsessive-compulsive disorder. Psychiatry Research: Neuroimaging, 198(1), 81-88. doi: 10.1016/j.psychres.2011.12.022

Simpson, H. B., Franklin, M. E., Cheng, J., Foa, E. B., \& Liebowitz, M. R. (2005). Standard criteria for relapse are needed in obsessive-compulsive disorder. Depression \& Anxiety, 21(1), 1-8. doi: 10.1002/da.20052

Simpson, H. B., Tenke, C. E., Towey, J. B., Liebowitz, M. R., \& Bruder, G. E. (2000). Symptom provocation alters behavioral ratings and brain electrical activity in obsessive-compulsive disorder: a preliminary study. Psychiatry Research: Neuroimaging, 95(2), 149-55. doi: 10.1016/ s0165-1781(00)00177-3
Steketee, G., Frost, R., \& Bogart, K. (1996). The Yale-Brown obsessive-compulsive scale: Interview versus self-report. Behavior Research \& Therapy, 34(8), 675-84. doi: 10.1016/00057967(96)00036-8

Storch, E. A., Larson, M. J., Price, L. H., Rasmussen, S. A., Murphy, T. K., \& Goodman, W. K. (2010). Psychometric analysis of the Yale-Brown obsessive-compulsive scale second edition symptom checklist. Journal of Anxiety Disorders, 24(6), 650-56. doi: 10.1016/j.janxdis.2010.04.010

Taylor, S. (2011). Early versus late onset obsessive-compulsive disorder: Evidence for distinct subtypes. Clinical Psychology Review, 31(7), 1083-100. doi: 10.1016/j.cpr.2011.06.007

Tot, S., Ozge, A., Comelekoglu, U., Yazici, K., \& Bal, N. (2002) Association of QEEG findings with clinical characteristics of OCD: Evidence of left frontotemporal dysfunction. Canadian Journal of Psychiatry, 47(6), 538-45.

Velikova, S., Locatelli, M., Insacco, C., Smeraldi, E., Comi, G., \& Leocani, L. (2010). Dysfunctional brain circuitry in obsessivecompulsive disorder: source and coherence analysis of EEG rhythms. NeuroImage, 49(1), 977-83. doi: 10.1016/j.neuroimage.2009.08.015

Wang, X., Cui, D., Wang, Z., Fan, Q., Xu, H., Qiu, J., et al. (2012). Cross-sectional comparison of the clinical characteristics of adults with early onset and late-onset obsessive compulsive disorder. Journal of Affective Disorders, 136(3), 498-504. doi: 10.1016/j.jad.2011.11.001

Wolpe, J., \& Lazarus, A. A. (1966). Behavior therapy techniques: A guide to the treatment of Neuroses. New York: Pergamon Press.

Yamanishi, T., Nakaaki, S., Omori, I. M., Hashimoto, N., Shinagawa, Y., Hongo, J., et al. (2009). Changes after behavior therapy among responsive and nonresponsive patients with obsessivecompulsive disorder. Psychiatry Research: Neuroimaging, 172(3), 242-50. doi: 10.1016/j.pscychresns.2008.07.004

Ye, C., An, C. X., \& Wang, X. Y. )2010). Impact of the biofeedback technology on OCD psychological and physiological parameters. Shanghai Archive Psychiatry, 18(5), 513-15. doi: 10.11919/j. issn.1002-0829.214067 